\title{
Endoleak after Endovascular Abdominal Aortic Aneurysm Repair Treated by Bilateral Transradial Access: Case Report
}

\author{
Gustavo A Samaja, Heber Tejerina Segovia, José M Escalante, Corina Biagioni and Silvana Onorato Grats \\ Interventional Cardiology Department, Policlinico Bancario Buenos Aires, Buenos Aires, Argentina
}

DOI: https://doi.org/10.17925/HI.2021.15.2.106

$\mathrm{T}$ he treatment of endovascular leaks after endovascular abdominal aortic repair can be challenging, particularly in patients with a lack of vascular access. We describe the case of a critically ill elderly patient with an endoleak resulting from structural failure of an endograft years after endovascular abdominal aortic repair. The patient was treated with an aorto-uni-iliac endoprosthesis, but a few days later a new endoleak appeared and femoral or axillar access was not feasible. We successfully treated the endoleak using a novel technique via bilateral transradial access involving simultaneous insufflation of two peripheral low-profile balloons to achieve a diameter capable of improving the apposition of the stent graft. In selected cases, bilateral radial access allows procedures to be performed that would otherwise be impossible due to the inherent limitation in sheath size that can be used in the radial artery.

\section{Keywords}

Endovascular aneurysm repair (EVAR) endoleak treatment, transradial interventions, case report, lack of vascular access, transradial peripheral interventions, abdominal aneurysm repair

Disclosures: Gustavo A Samaja, Heber Tejerina Segovia, José M Escalante, Corina Biagioni and Silvana Onorato Grats have no financial or non-financial relationships or activities to declare in relation to this article.

Review process: Double-blind peer review.

Compliance with ethics: All procedures were followed in accordance with the responsible committee on human experimentation and with the Helsinki Declaration of 1975 and subsequent revisions, and informed consent was received from the patient involved in this case study.

Data availability: Data sharing is not applicable to this article as no datasets were generated or analysed during the writing of this article.

Authorship: The named authors meet the International Committee of Medical Journal Editors (ICMJE) criteria

for authorship of this manuscript, take responsibility

for the integrity of the work as a whole, and have given final approval for the version to be published.

Access: This article is freely accessible at

touchCARDIO.com. (C) Touch Medical Media 2021

Received: 24 September 2021

Accepted: 16 November 2021

Published online: 20 December 2021

Citation: Heart International. 2021;15(2):106-11

Corresponding author: Gustavo A Samaja,

Policlinico Bancario Buenos Aires, Gaona

2195, Ciudad de Buenos Aires, CABA 112,

Argentina.E.gustavosamaja@hotmail.com

Support: No funding was received for

the publication of this article.
A common complication of endovascular repair of an abdominal aortic aneurysm (EVAR) is the persistence of blood flow outside the lumen of the endoprosthesis, known as an endoleak. Endoleaks are a potentially lethal complication and occur following up to $25 \%$ of EVAR procedures. ${ }^{1,2}$ The classification of endoleaks includes five categories according to blood flow into the aneurysm sac (Figure 1A). ${ }^{3}$ Type I and type III endoleaks represent direct communication with the systemic blood flow and the aneurysm sac, and require immediate repair. The treatment depends on the type of endoleak, and can be as simple as balloon angioplasty, or much more complex such as the embolization of lumbar or inferior mesenteric branches, or even placement of a new stent graft or open repair (Figure 1B). ${ }^{4}$ We describe a patient with a type I endoleak, which occurred a few days after treatment for a type IIIB endoleak of a bifurcated stent graft that had been implanted years earlier. A lack of femoral or axillar access in the patient meant that endovascular treatment was the only reasonable option. We used a novel technique involving bilateral transradial access (TRA) and balloon angioplasty by simultaneous insufflation of two peripheral non-compliant balloons to achieve appropriate apposition of the recently implanted endograft.

\section{Case description}

An 82-year-old man affected by coronary disease, chronic obstructive pulmonary disease, hypertension and dyslipidaemia was admitted to the intensive care unit with pneumonia and cardiac failure. He had undergone EVAR several years earlier with placement of an Endologix AFX® bifurcated stent graft (Endologix LLC, Irvine, CA, USA).

Cardiac failure improved with standard treatment, but pneumonia did not respond to antibiotic therapy. A few days after admission, the patient suddenly developed abdominal pain radiating to the back, a tender abdominal mass and drop in haematocrit. Computed tomography (CT) angiography scan showed the presence of a large type III endoleak arising from the main body of the endograft, and signs of aortic rupture with retroperitoneal and perirenal haematoma (Figure 2). Angiography confirmed the large type III endoleak at the level of the body of the endograft, with aortic rupture (Figure 3).

The patient underwent successful positioning of an aorto-uni-iliac endograft. The Hercules ${ }^{\mathrm{TM}}$ endograft (MicroPort Scientific Corp., Shanghai, China) has a tapered diameter, $30 \mathrm{~mm}$ to $18 \mathrm{~mm}$, and length of $105 \mathrm{~mm}$, and was deployed within the body of the Endologix stent graft, extending across the defect and terminating in the right limb. Next, occlusion of the contralateral limb was performed using a plug. Finally, a femoro-femoral crossover bypass graft was created. The procedure was considered successful, despite suboptimal findings on angiography after endograft deployment (Figure 4). 


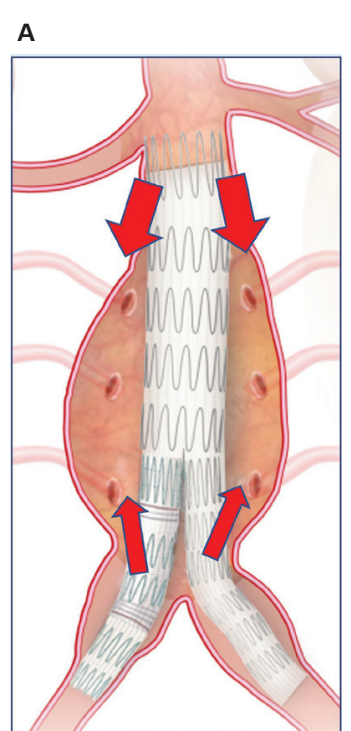

Type I

Proximal or distal graft attachment site leaks

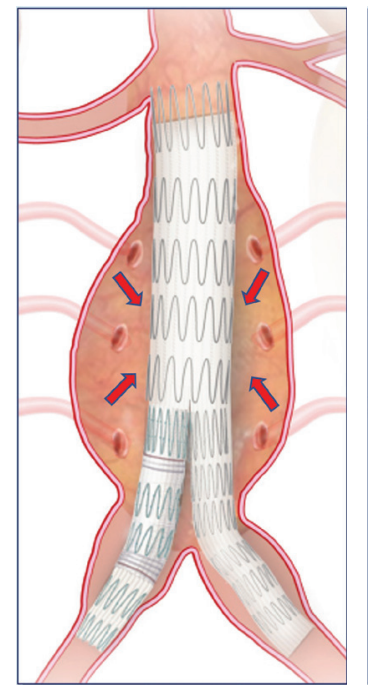

Type II

Retrograde flow into the aneurysm sac from aortic side-branches such as the lumbar or inferior mesenteric arteries

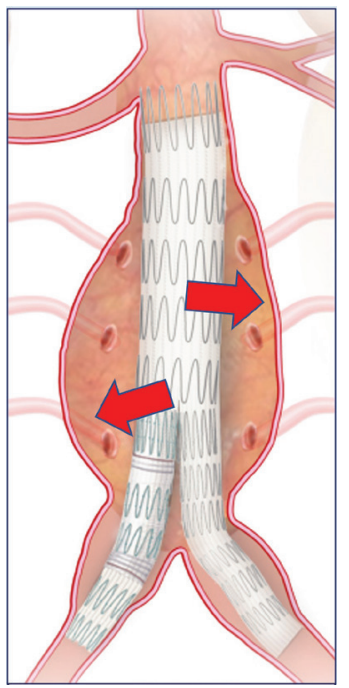

Type III

Caused by a defect in the graft either due to a fabric tear or disconnection of modular overlap

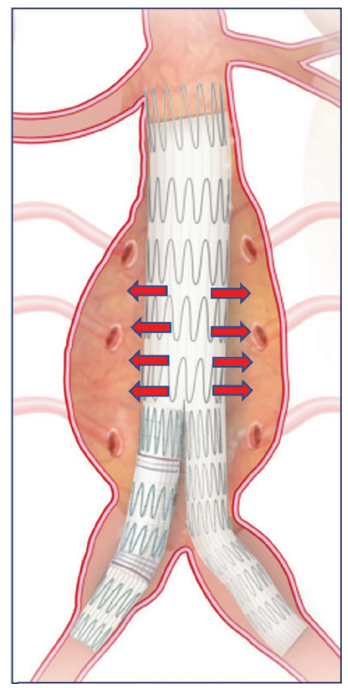

Type IV

Graft wall porosity

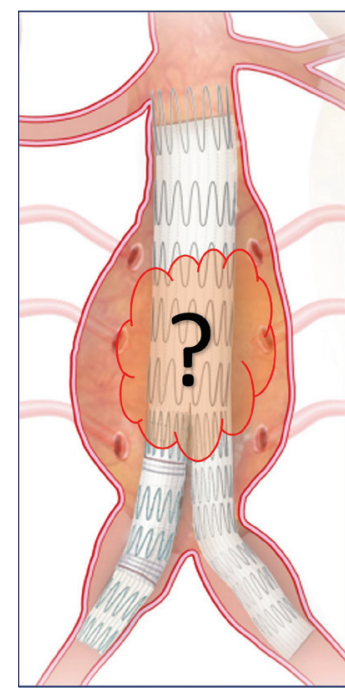

Type $\mathbf{V}$

Increase in maximum aneurysm diameter with no identifiable endoleaks

B

\section{Endovascular treatment}

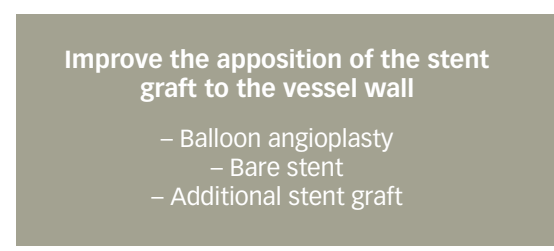

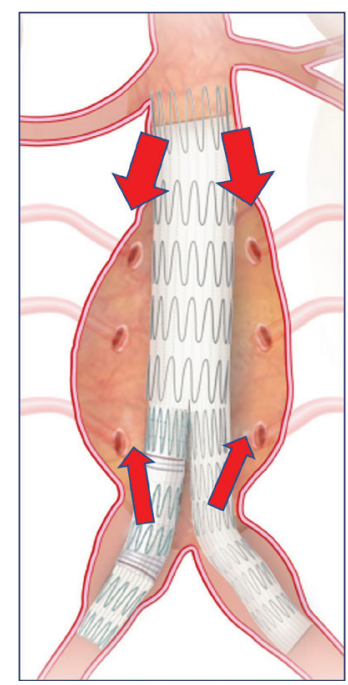

Type I

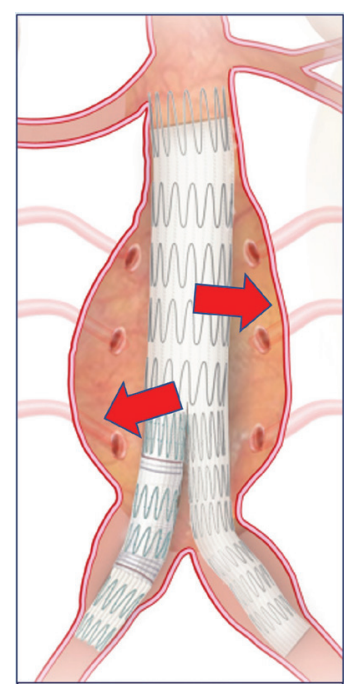

Type III

A: types of endoleak. There are four types of endoleaks, and a proposed but controversial fifth type; B: treatment of type I and III endoleaks.

The patient had a poor clinical evolution, including poor response to antibiotic therapy, drop in platelet count, renal impairment and general deterioration. Four days after the procedure, the patient presented sudden onset of abdominal pain and tenderness, and had a new drop in haematocrit. A CT angiography scan showed an endoleak at the level of the aortic bifurcation (Figure 5).

The patient's situation was critical. He had an endoleak requiring emergency treatment, ideally without deploying a third endograft because of the active infection. Open repair was not an option, so a new endovascular treatment was needed; however, the patient had a lack of access sites. Transaxillary access was not reasonable because the platelet count was low; left femoral access was not available because of the plug in the left iliac artery; and right femoral artery access was a bad option because it had been dissected to create the femoro-femoral bypass.

In consultation with the interventional cardiology team, we proposed a previously undescribed approach for treating the patient: bilateral TRA, with the following rationale. We expected a type I endoleak, and suspected poor apposition between the distal attachment site of the newly deployed stent graft and the previous stent graft. Ideally, angioplasty should resolve the endoleak. Stents, extender cuffs or a third endograft should be avoided because of the active infection. Angioplasty should be performed with an $18-20 \mathrm{~mm}$ diameter balloon, 
Figure 2: Computed tomography angiography scan following symptoms of abdominal pain, tender abdominal mass and drop in haematocrit

A

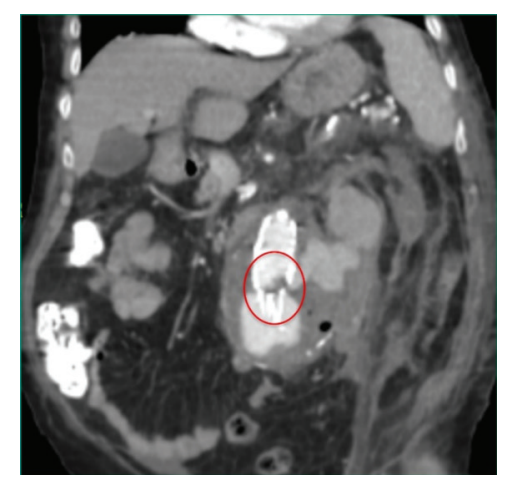

B

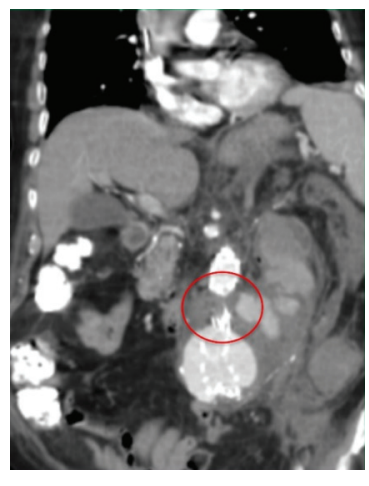

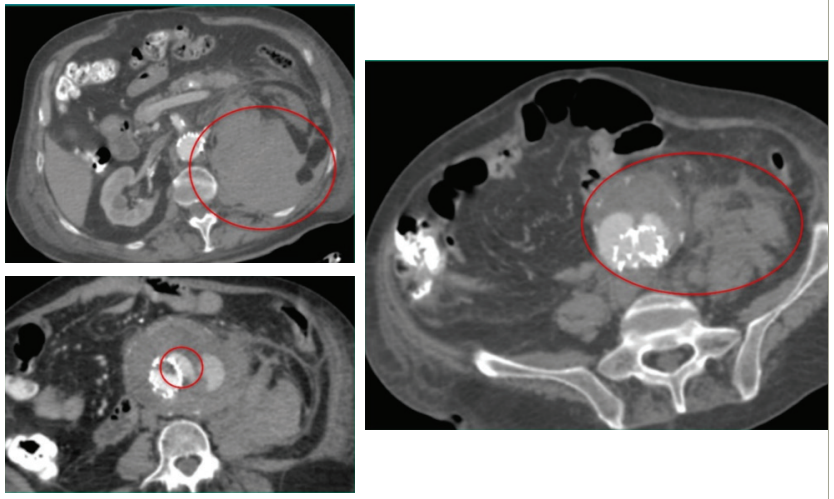

A: endoleak at the level of the main body of the endograft (circled); B: there were signs of aortic rupture with retroperitoneal and perirenal haematoma (circled).

Figure 3: Angiography following symptoms of abdominal pain, tender abdominal mass and drop in haematocrit
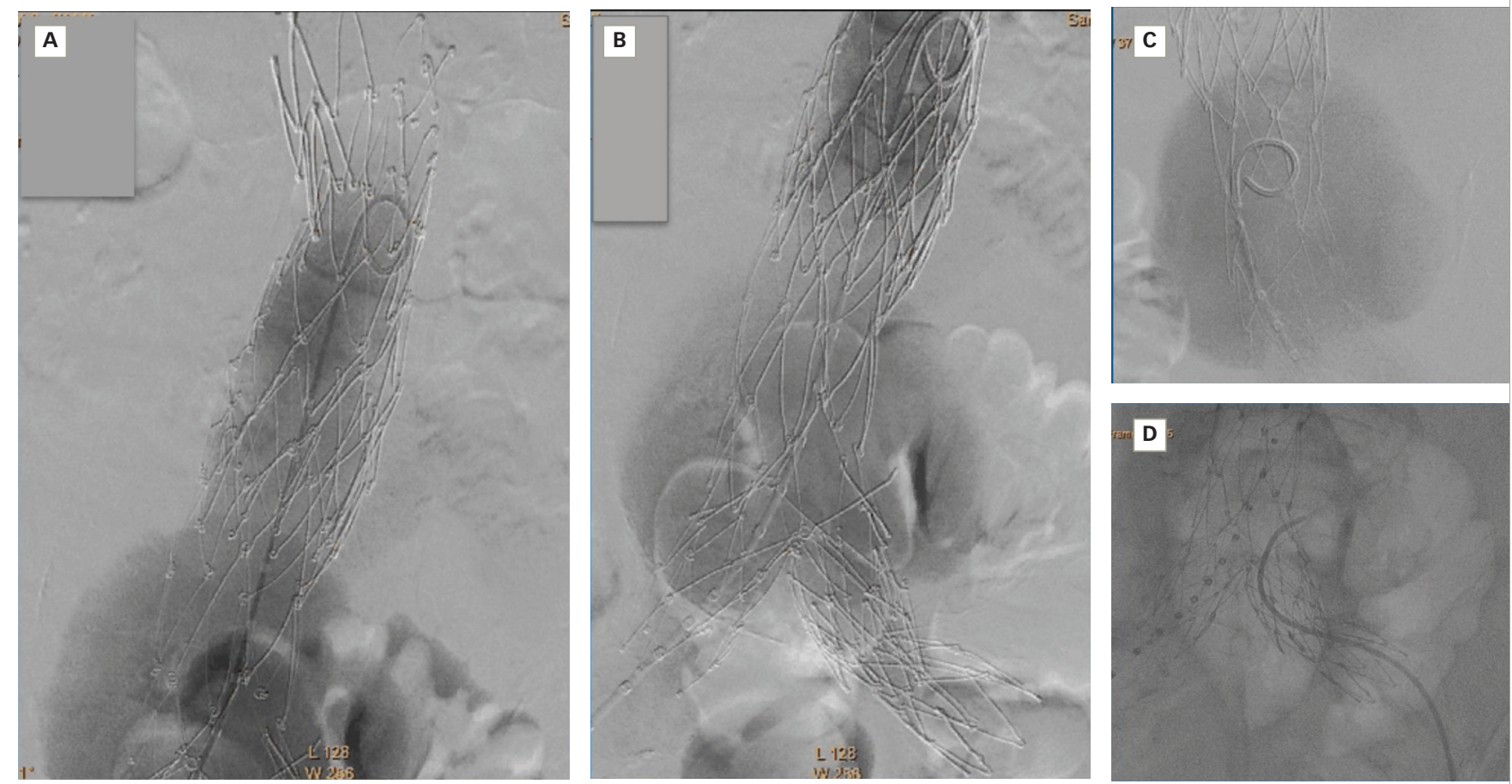

A-D: large type III endoleak at the level of the body of the bifurcated endograft, with aortic rupture.

but this is impossible via TRA due to limitations in the sheath size that can be used in the radial artery. Nevertheless, if bilateral TRA was successful, we could simultaneously insufflate two balloons at the level of the leak, one through each arm. We could employ up to two $10 \mathrm{~mm}$ diameter balloons, as they can be deployed through a $6 \mathrm{Fr}$ sheath; if necessary, we could use up to two $12 \mathrm{~mm}$ diameter balloons by upsizing to $7 \mathrm{Fr}$ sheaths.

We performed an angiography via right TRA. We used a pigtail catheter and then a multipurpose catheter. Aortography at the level of the renal arteries did not show the leak; consequently, we presumed a type IB or type III endoleak. The leak was found at the level of the aortic bifurcation, precisely in the proximal segment of the right limb of the Endologix endograft (Figure $6 A$ ). This segment was not covered by the recently deployed stent graft, so we hoped that the leak was related to misalignment and not to a tear in the graft, which would have required a third endograft.

We decided to try to seal the defect using balloon angioplasty. The first step involved left TRA and angiography, then right TRA and positioning two extra stiff guidewires into the right limb of the Endologix, one through the right radial access and the other through the left radial access. Our plan was to seal the distal segment of the recently deployed stent graft, and then to proceed towards the limb of the Endologix with the aid of balloon insufflations. We used two $8 \times 40 \mathrm{~mm}$ and then two $10 \times 40 \mathrm{~mm}$ peripheral balloons (Mustang; Boston Scientific, Marlborough, MA, USA), calculating that the combined diameter ( $20 \mathrm{~mm}$ plus) should be sufficient to seal a distal leak. Recommended sheath size for $10 \mathrm{~mm}$ peripheral 


\section{Figure 4: Angiography after endograft deployment}

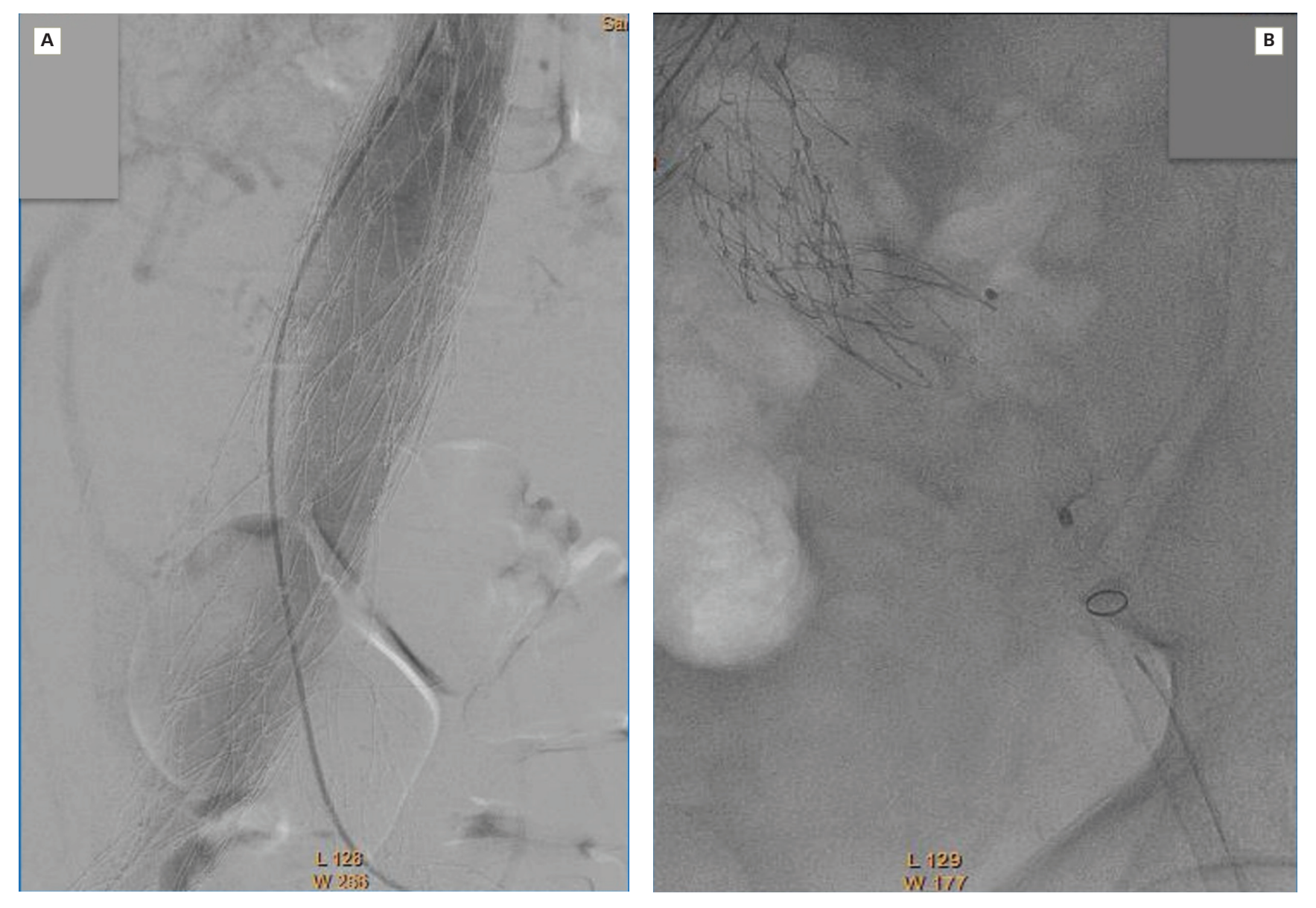

A: Hercules endograft (MicroPort Scientific Corp., Shanghai, China); B: Vascular plug.

Figure 5: Computed tomography angiography scans, showing the endoleak at the level of the aortic bifurcation (circled)
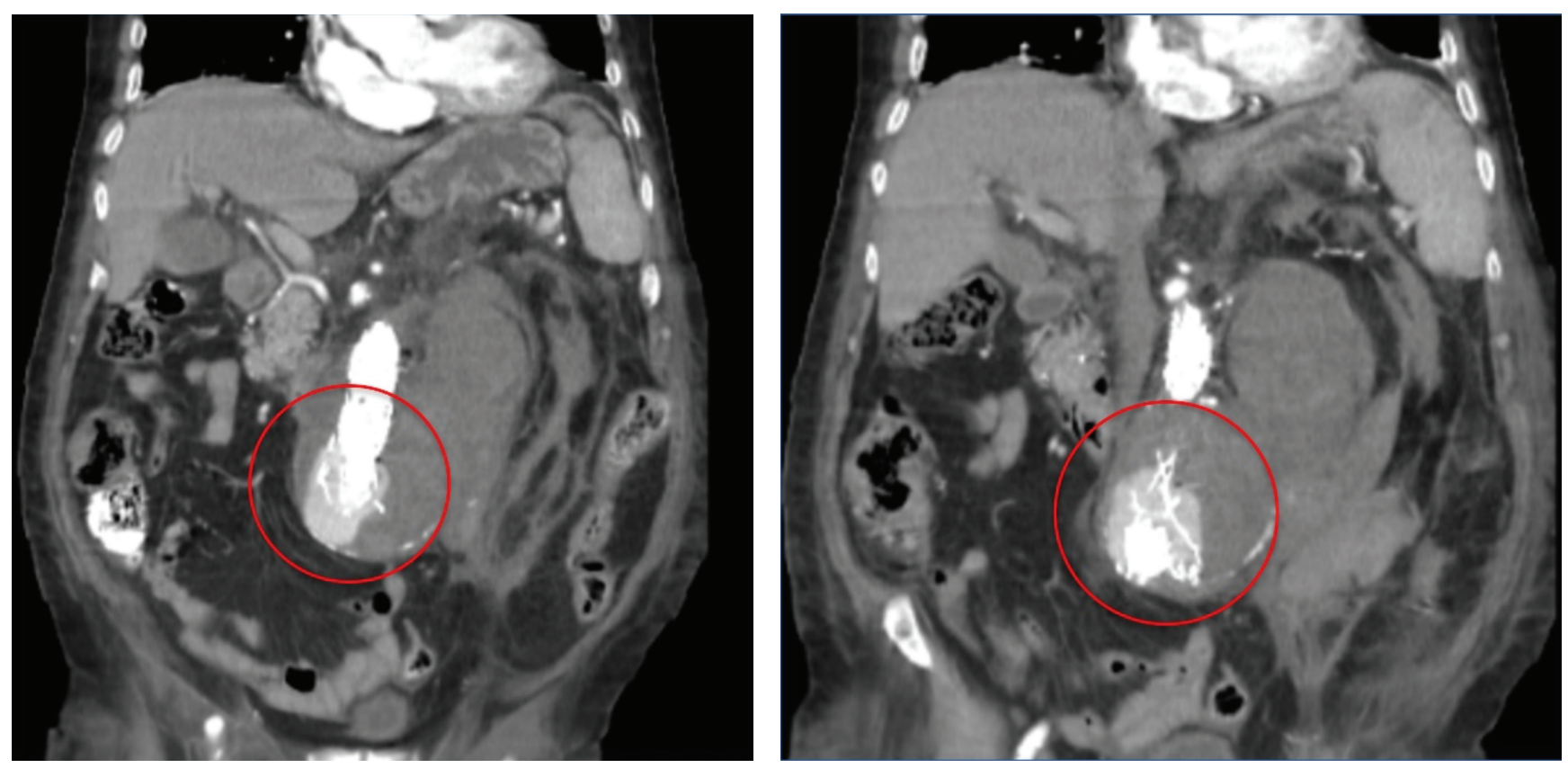


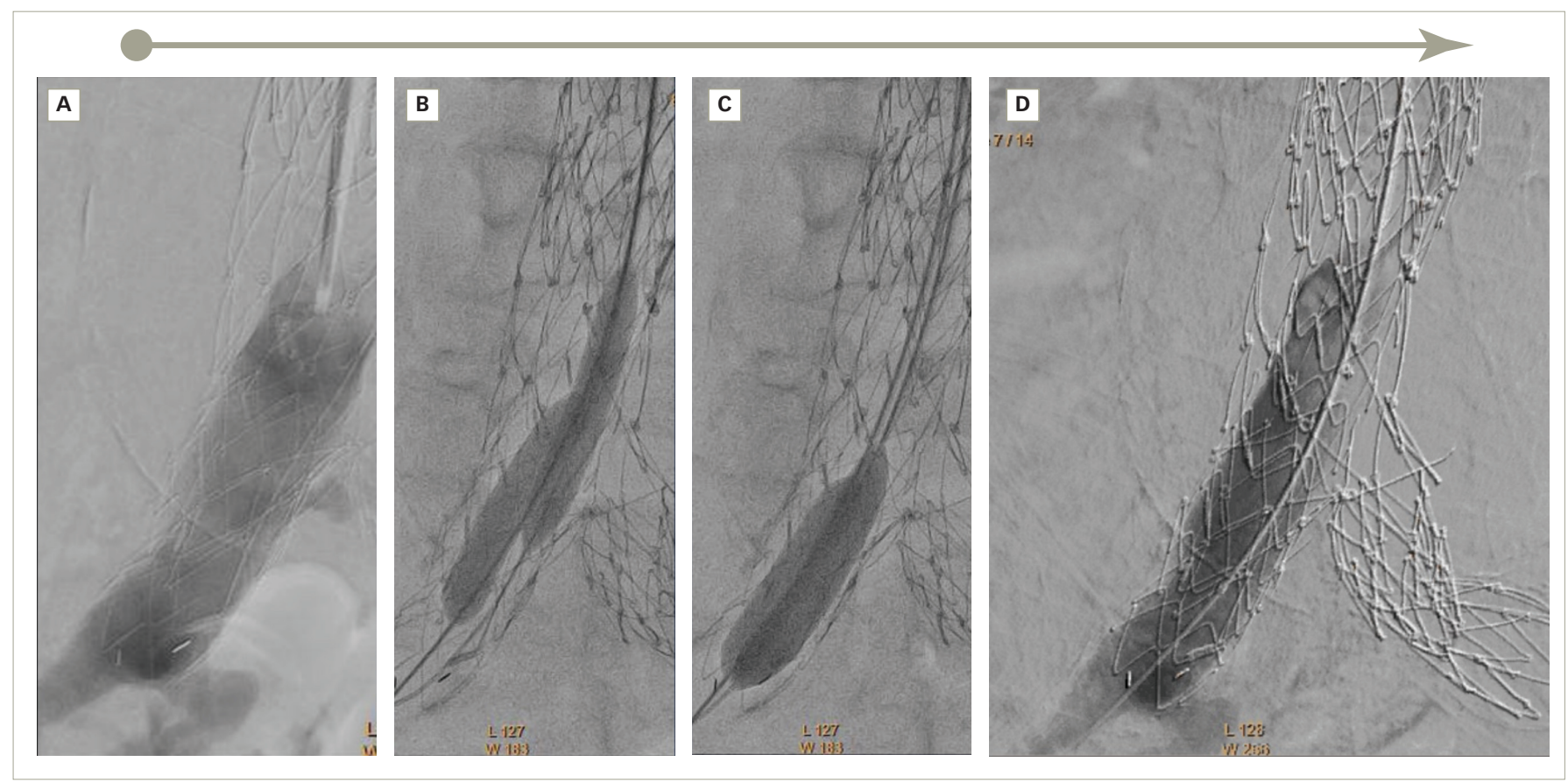

A: Pre-procedure angiography; B: Simultaneous insufflation of two $8 \times 40 \mathrm{~mm}$ peripheral balloons; C: Simultaneous insufflation of two $10 \times 40 \mathrm{~mm}$ peripheral balloons; D: Final angiogram, showing the sealed leak.

balloons is $6 \mathrm{Fr}$. We introduced the balloons through the sheaths and advanced the balloons over the extra stiff guidewires ("bare on the wire" technique). We performed several simultaneous low-pressure insufflations within the aortic bifurcation and the right limb of the stent graft (Figure $6 B-C$ ). The leak was sealed, and no additional manoeuvre was required (Figure 6D).

The patient recovered well, after a long hospital stay related to his comorbidities. He lost the left radial pulse, but this could have been related to an arterial line that he had in his left wrist for 1 week after our procedure. There was no hand ischaemia or dysfunction. At 4 months' follow-up, CT angiography confirmed endograft and bypass patency, and no endoleak. The late follow-up was uneventful, with no clinical evidence of complications or leak at 18 months.

\section{Discussion}

We think that this is an interesting learning case, illustrating points of therapeutic strategy that could be improved and providing an elegant resolution using a previously undescribed technique.

The first point is a concern about the first procedure that we performed to treat the endoleak, which certainly could have been improved. We must suspect that the second leak was already there after the first procedure, as the final angiogram was not optimal. Besides, an elastomeric balloon for moulding the aorta was not employed as the last step of the procedure; we think that this should have been used on a routine basis when deploying a second endograft inside an existing endograft.

The nature of the second leak presented a dilemma. It looked like a type III endoleak, which implies holes, defects or separations in the stent graft material and requires the placement of a new stent graft component across the defect or junctional separation. However, it was successfully treated as if it was a type I endoleak because balloon angioplasty was successful. Our explanation is that it was a leak at the distal attachment site (type IB endoleak) in an endograft deployed for the treatment of a type III endoleak. Consequently, we sealed the type III endoleak of the Endologix bifurcated stent graft implanted years ago when we resolved the type I endoleak of the Hercules endograft deployed recently.

Regarding the Endologix endograft, in 2015 the manufacturers reported data from a 5-year follow-up, showing no type III or IV endoleaks. ${ }^{5}$ Concerns appeared later, however, and in a document released in December 2020, the US Food and Drug Administration confirmed the previous recommendation of at least yearly, lifelong follow-up with the use of the Endologix endograft system because of the risk of type III endoleaks. ${ }^{.}$

Low-pressure insufflation balloon angioplasty of the affected attachment site is the accepted first-line treatment for type I endoleaks by moulding the aorta. This is usually done with large-diameter balloons to achieve a 1:1 balloon:artery ratio with the abdominal aorta or iliac artery. In this patient, the problem was the lack of vascular access, and we employed a previously undescribed but very simple strategy to achieve angioplasty via TRA: simultaneous insufflation of two peripheral balloons, one inserted from the left and the other from the right arm. With two balloons simultaneously insufflated, we achieved the necessary diameter for treating the endoleak. We applied low-pressure, manual insufflation as the Mustang peripheral balloons are non-compliant, and the aim was to mould the aorta not to treat an obstruction.

The simultaneous insufflation of two $10 \mathrm{~mm}$ peripheral balloons sealed the distal attachment site as if a 19-20 mm moulding balloon had been used, but we must keep in mind two technical aspects. First, dual balloon inflations cause elliptical/oval expansion of the graft. Consequently, depending on the orientation of the balloons, if the maximal expansion diameter is not located at the site of the graft malapposition, the manoeuvre may not adequately remedy the leak. Second, the maximal diameter achieved is not the simple mathematical addition of the balloon diameters, and must be calculated using the Mitsudo formula; 7 however, as we were moulding the aorta in this case rather than treating stenotic disease, this might not be a critical issue. 


\section{Conclusions}

Aortic stent graft endoleak was successfully treated via bilateral TRA followed by simultaneous insufflation of two peripheral balloons, one deployed from the right arm and the other from the left arm. In selected

cases, bilateral access allows transradial procedures to be performed that would otherwise be impossible due to the inherent limitation of sheath size. $\square$

1. Golzarian J, Maes EB, Sun S. Endoleak: treatment options. Tech Vasc Interv Radiol. 2005;8:41-9.

Hellinger JC. Endovascular repair of thoracic and abdominal aortic aneurysms: pre- and postprocedural imaging. Tech Vasc Interv Radiol. 2005;8:2-15.

Görich J, Rilinger N, Sokiranski R, et al. Leakages after endovascular repair of aortic aneurysms: classification based on findings at CT, angiography, and radiography. Radiology. 1999;213:767-72.

White SB, Stavropoulos SW. Management of endoleaks following endovascular aneurysm repair. Semin Intervent Radiol. 2009;26:33-8.
Endologix. 2016-2019 clinical update - AFX® endovascular AAA system. Available at: https://endologix.com/wp-content/uploads/2019/10/MM2165-Rev-01-Endologix-2016-2019-AFX-Clinical-Update.pdf Endologix. 2016-2019 clinical

6. US Food and Drug Administration. FDA safety communication. Update: the FDA reminds patients and health care providers of the importance of at least yearly, lifelong follow-up with use of Endologix AFX endovascular AAA graft systems. Available at: www.fda.gov/medical-devices/safety-communications/update-fda-reminds-patients-and-health-care-providers-importance-least-yearly-lifelong-follow-use (accessed 4 December 2020).

7. Morino $Y$, Yamamoto $\mathrm{H}$, Mitsudo $\mathrm{K}$, et al. Functional formula to determine adequate balloon diameter of simultaneous kissing balloon technique for treatment of bifurcated coronary lesions clinical validation by volumetric intravascular ultrasound analysis. Circ J. 2008;72:886-92. 